\title{
METODOLOGIA BOX \& JENKINS E ANÁLISE DE DADOS EM PAINEL NA PREVISÃO DE SÉRIES FINANCEIRAS
}

\author{
Nayane Thais Krespi \\ Universidade Federal do Paraná - UFPR \\ nkrespi@gmail.com \\ Anselmo Chaves Neto \\ Universidade Federal do Paraná - UFPR \\ anselmo@ufpr.br
}

\section{RESUMO}

Este estudo tem como objetivo avaliar qual o melhor método de previsão do preço das ações da carteira teórica composta pelas empresas integrantes do $\mathrm{IBrX}-50$, análise de séries temporais ou análise de dados em painel. Em relação aos procedimentos metodológicos foram pesquisadas 23 empresas componentes do índice IBrX-50 listadas na BM\&FBovespa desde ao menos 2014. A variável utilizada em todas as análises foi o preço de fechamento da ação do último dia de cada mês (portanto dados mensais) e o período de análise corresponde aos anos de 2004 até 2014, sendo 10 anos (2004-2013) para análise e 2014 para realizar as previsões. Com a previsão pelo modelo de séries temporais, encontrou-se uma única empresa que apresentou erro quadrático médio inferior nesse modelo, a empresa KLABIN S/A. Já a empresas USIMINAS evidenciou erro quadrático médio zerado para ambos os modelos analisados. Nos demais casos estudados, ou seja em 92,30\% das empresas pesquisadas, o modelo de previsão por dados em painel se mostrou mais preciso em relação a previsão por séries temporais.

Palavras-chave: Séries temporais. Dados em Painel. Preço de ações. Previsão.

\begin{abstract}
This study aims to evaluate what the best price forecasting method of the shares of hypothetical portfolio of companies of the IBrX-50, time series analysis and panel data analysis. Regarding the methodological procedures were surveyed 23 companies participating in the IBrX-50 index listed on the BM \& FBovespa since at least 2014. The variable used in all analyzes was the share closing price of the last day of each month (hence monthly data) and the analysis period corresponds to the years 2004 until 2014, 10 years (2004 to 2013) for consideration and 2014 to make forecasts. With the forecast for the time series model, we found one company that had a mean square error lower in this model, the Klabin S / A. Have Usiminas companies showed mean square error reset for both analyzed models. In the other cases studied, ie in $92.30 \%$ of the companies surveyed, the data for forecasting model panel was more precise about the forecast by time series.
\end{abstract}

Keywords: Time series. Panel Data. Stock price. Forecast. 


\section{INTRODUÇÃO}

O desenvolvimento das sociedades está intimamente relacionado com o mercado financeiro, visto que é por meio de seus componentes que a transferência de recursos é viabilizada. Os mercados acionários constituem uma subdivisão importante do mercado de capitais, pois executam tarefas vitais na economia, como por exemplo auxílio no processo de alocação de recursos financeiros, oportunidades de investimentos para agentes superavitários, e oportunidades de obtenção de capital para empresas que têm projeto de investimento com valor presente líquido positivo (BRUNI, 1998).

A forma de ação dos investidores, bem como o funcionamento do mercado acionário sempre foram relevantes e constantes temas de pesquisa. Nesse sentido, a Teoria de Finanças sofreu alterações profundas com o passar do último meio século. Nesse processo de evolução, a teoria em questão incorporou técnicas e expressões exóticas, aparentemente incomum ao mercado financeiro, como por exemplo: redes neurais, teoria do caos, fractais e algoritmos genéticos. Sendo assim, observa-se que as ciências exatas, como a matemática e a física, ligamse cada vez mais aos estudos financeiros (BRUNI, 1998).

No mercado de capitais, as ações proporcionam aos investidores participação proporcional no fluxo de caixa da empresa, ou seja, a empresa se compromete a pagar, de forma periódica, dividendos proporcionais ao seu portador. Este mercado é interessante para a empresa, visto que a movimentação de ações no mercado (emissão e venda) permite que esta capte recursos financeiros e aplique em investimentos produtivos (ANTUNES; PROCIANOY, 2002).

Logo, o objetivo deste artigo é avaliar qual o melhor método de previsão do preço das ações da carteira teórica composta pelas empresas integrantes do $\mathrm{IBrX}-50$, análise de séries temporais ou análise de dados em painel.

\section{CARTEIRA DE AÇÕES}

O mercado financeiro tem grandes preocupações com a maneira como um investidor poderia decidir da melhor forma possível sua composição de investimentos, analisando em relação aos retornos esperados e aos riscos incorridos. O investidor sempre busca maximizar os retornos e minimizar os riscos, o que torna essa a decisão ótima (KRITZMAN, 1992).

A forma de composição dos investimentos é realizada entre escolhas acerca do risco e do retorno, ou seja, um investidor pode escolher entre vários conjuntos de diferentes ações, denominado carteira de ações. Portanto, o investidor pode decidir como aplicar seus recursos, distribuindo-os entre diferentes ativos de retornos e riscos desiguais. (BRUNI, 1998). Dessa forma, Sharpe, Alexander, Bailey (1995) definem carteira de ações (ou carteira de mercado) como um portfólio formado por todos os ativos no qual a proporção investida em cada ativo corresponde ao seu valor relativo de mercado.

A partir deste momento, vale definir a teoria desenvolvida por Markowitz designada por Moderna Teoria de Portfólios. Esta teoria, segundo o próprio Markowitz (1952), apresenta o risco como fator inerente às decisões de investimento, de forma a contrariar o senso comum, que é concentrar os recursos em um único ativo de maior retorno esperado. Define-se portfólio como "uma carteira de títulos que contém ações, obrigações, mercadorias, investimentos em imóveis, investimentos em títulos de liquidez imediata ou outros ativos de um investidor pessoa física ou institucional." (DOWNES; GODMAN, 1993, p. 385).

Ainda segundo Markowitz (1952), a principal conclusão que se obtém a partir de sua teoria é que um investidor, ao analisar um ativo, não deve estar preocupado somente com o seu risco individual, mas sim, na contribuição deste ao risco total do portfólio. Essa é a ideia de diversificação, introduzida pelo autor, e até aquele momento, contestada por pensadores influentes da época. Por volta de 20 anos depois da publicação de seu trabalho Markowitz praticamente extinguiu as concepções ingênuas acerca da diversificação, mostrando que não 
"bastava colocar os ovos em vários cestos diferentes e, quanto maior o número de cestos, maior a segurança." (BRUNI, 1998, p. 44).

Conforme as ideias expostas por Markowitz (1952) uma diversificação feita de forma apropriada e correta pode reduzir os riscos inerentes ao mercado acionário. Um exemplo simples dessa ideia é, se aumentar o preço do petróleo prejudica os resultados de uma empresa de aviação, o investidor poderia reduzir esse risco (exposto no preço da ação), por meio da compra de ações de uma empresa petrolífera (a qual teria melhora nos seus resultados pelo fato do preço do petróleo estar em alta).

\section{SÉRIES TEMPORAIS - METODOLOGIA BOX \& JENKINS}

Sabe-se que modelo ARIMA e modelo Box \& Jenkins são denominações sinônimas. Três etapas, realizadas por meio de um processo iterativo, podem ser utilizadas na construção de modelos ARIMA, sendo: identificação do modelo; estimação dos parâmetros do modelo; e verificação do modelo (MORETTIN; TOLOI, 2006).

Os dados que compõem a série temporal são analisados e dessa forma, procede-se a identificação do modelo ARIMA a ser utilizado. (BOX; JENKINS; REINSEL, 2008). Existem diversos modelos ARIMA, o que deixa que identificação do modelo a ser utilizado não seja uma tarefa trivial.

Com o modelo já identificado, é necessário estimar seus parâmetros. Para a estimação dos parâmetros de modelos ARIMA, o método dos mínimos quadrados pode ser utilizado. Contudo, para os componentes MA, não existe uma maneira simples de proceder a estimativa dos parâmetros (MAKRIDAKIS; WHEELWRIGHT; HYNDMAN, 1998). Ainda no que tange a estimação dos parâmetros, outro método bastante utilizado é o da máxima verossimilhança.

Após obter um modelo ajustado para a série temporal, precisa-se determinar a sua adequação e qual a necessidade de melhoria. Existe um método lógico que executa a verificação do modelo e utiliza o cálculo de resíduos $\left(e_{t}=z_{t}-\hat{z}_{t}\right)$. Portanto, precisa-se examinar a função de autocorrelação dos resíduos (BOX; JENKINS; REINSEL, 2008).

Caso o modelo obtido seja adequado, a FAC da amostra dos resíduos $r_{e}(k)$ não deve apresentar lags significativos para nenhum valor de $\mathrm{k}$, que para este caso foram definidos com sendo os maiores que $n^{-1 / 2}$. Todavia, se este for o caso, os valores observados na série temporal $z_{t}$ foram transformados em um processo de ruído aleatório $e_{t}$.

Os testes de Portmanteau são outra forma de verificar a significância. Ao contrário do teste anterior, este não considera os valores de $r_{e}(k)$ individualmente, mas sim o conjunto dos $\mathrm{k}$ primeiros $r_{e}(k)$ 's. Estes testes apresentam (aproximadamente) uma distribuição Quiquadrado e testam a hipótese de um conjunto de resíduos ser significativo. Os testes de Portmanteau não são muito precisos, e portanto, devem ser utilizados apenas como um teste auxiliar (MAKRIDAKIS; WHEELWRIGHT; HYNDMAN, 1998).

Neste ponto da análise, já se conhece o melhor modelo para a série temporal em análise e, pode-se utilizá-lo com o intuito de gerar previsões de observações futuras. Ou seja, parte-se do período atual $t$, e supõe-se que se deseja prever a série em um período futuro, $\hat{z}_{t+k}$. Esta nova série representa a previsão para um período $\mathrm{t}+\mathrm{k}$ feita em $\mathrm{t}$ (BOX; JENKINS; REINSEL, 2008).

\section{ANÉLISE DE DADOS EM PAINEL}

O modelo de dados em painel envolve, em sua análise, dados provenientes de várias cross-sections ao longo do tempo. Em outras palavras, é um modelo capaz de analisar simultaneamente diversas informações em vários períodos de tempo (FÁVERO et al, 2014). É importante salientar que a principal vantagem da análise de dados em painel, apontada por Marques (2000), é a possibilidade de controlar a heterogeneidade individual, que em outras 
palavras quer dizer, a possibilidade de se medir separadamente os efeitos gerados por conta de diferenças existentes em cada observação em cada cross-section.

A diversidade de modelos de dados em painel existentes atualmente é vasta. Todavia, a divisão básica entre esses modelos se dá separando-os em dois grupos: i) modelos de efeitos fixos; e ii) modelos de efeitos aleatórios. Apesar da nomenclatura, tanto os modelos de efeitos fixos quanto os modelos de efeitos aleatórios possuem efeitos, no nível individual (empresas), aleatórios (GREENE, 2007).

O modelo geral que descreve os dados em painel é dado por:

$$
y_{i t}=\beta_{0 i t}+\beta_{1 i t} x_{1 i t}+\cdots+\beta_{\text {nit }} x_{k i t}+\varepsilon_{i t}
$$

onde: $i$ denota os diferentes indivíduos;

$t$ denota o período de tempo que está sendo analisado;

$\beta_{0}$ refere-se ao parâmetro de intercepto; e modelo.

$\beta_{k}$ refere-se ao coeficiente angular correspondente a k-ésima variável explicativa do

A forma matricial do modelo é dada por:

$$
\begin{aligned}
y_{i}=\left[\begin{array}{c}
y_{i 1} \\
y_{i 2} \\
\vdots \\
y_{i T}
\end{array}\right] ; X_{i} & =\left[\begin{array}{cccc}
x_{1 i 1} & x_{2 i 1} & \cdots & x_{K i 1} \\
x_{1 i 2} & x_{2 i 2} & \ldots & x_{K i 2} \\
\vdots & \vdots & \ddots & \vdots \\
x_{1 i T} & x_{2 i T} & \ldots & x_{K i T}
\end{array}\right] ; \beta_{i}=\left[\begin{array}{ccccc}
\beta_{0 i 1} & \beta_{1 i 1} & \beta_{2 i 1} & \ldots & \beta_{K i 1} \\
\beta_{0 i 2} & \beta_{1 i 2} & \beta_{2 i 2} & \ldots & \beta_{K i 2} \\
\vdots & \vdots & \vdots & \ddots & \vdots \\
\beta_{0 i T} & \beta_{1 i T} & \beta_{2 i T} & \ldots & \beta_{K i T}
\end{array}\right] ; \varepsilon_{i} \\
& =\left[\begin{array}{c}
\varepsilon_{i 1} \\
\varepsilon_{i 2} \\
\vdots \\
\varepsilon_{i T}
\end{array}\right] \quad(02)
\end{aligned}
$$

onde: $y_{i}$ e $\varepsilon_{i}$ são vetores de dimensão ( $\mathrm{T}$ x 1) e contém, respectivamente, as $\mathrm{T}$ variáveis dependentes e os T erros;

$X_{i}$ é uma matriz de dimensão ( $\mathrm{K}$ x T $\mid$ ) com as variáveis explicativas do modelo;

$\beta_{i}$ é a matriz dos parâmetros a serem estimados.

É fácil verificar que, para este modelo, o intercepto e os parâmetros resposta diferem para cada indivíduo e para cada período de tempo. Sendo assim, tem-se mais valores desconhecidos do que observações, o que torna impossível estimar esses parâmetros. Para tanto, faz-se necessário especificar suposições acerca do modelo geral, (01) ou (02), com o intuito de torná-lo estimável operacionalmente (DUARTE; LAMOUNIER; COLAUTO, 2008).

Ainda segundo os autores supra citados, existem três modelos que são os mais utilizados quando se trata de mesclar dados de séries temporais e dados de corte transversal. São eles: i) Modelo de Regressões Aparentemente Não-Relacionadas; ii) Modelos de Efeitos Fixos; e iii) Modelos de Efeitos Aleatórios.

\section{PROCEDIMENTOS METODOLÓGICOS}

A população de pesquisa compreende todas as empresas que compunham o índice IBRX-50 da BM\&FBovespa no $1^{\circ}$ quadrimestre (janeiro - abril) de 2015 , perfazendo um total de 50 empresas. Integram esse grupo empresas pertencentes aos diversos setores econômicos da bolsa.

Conforme a própria definição do índice IBRX-50, 50 empresas compõem a população da presente pesquisa. Por sua vez a amostra da pesquisa é composta por 23 empresas, as demais empresas foram retiradas da amostra por falta de dados disponíveis para análise. 
Os dados utilizados na presente pesquisa foram obtidos por meio da base de dados Thomson. Para alguns esclarecimentos adicionais, foram também consultadas às demonstrações contábeis disponibilizadas no endereço eletrônico da BM\&FBovespa. Por fim, o período de análise da variável de pesquisa é de 11 anos, iniciando em 2004 e findando em 2014. Dessa forma, cada empresa analisada terá 132 dados coletados, sendo 12 de cada ano multiplicado por 11 anos.

Além disso, para proceder a análise clássica de dados em painel, necessita-se de uma variável dependente e ao menos uma variável independente. No caso do presente estudo, temse apenas uma variável em análise, preço de ação. Para resolver esse impasse, recorreu-se ao modelo de Koyck, cuja ideia é utilizar um dado passado na equação atual, em outras palavras, no modelo original a variável dependente $y_{t-1}$ passa a ser uma variável independente no momento que calcula-se $y_{t}$.

$\mathrm{Na}$ adaptação proposta, a ideia é justamente considerar $y_{t-1}$, que seria uma variável dependente, como uma variável independente no modelo em que $y_{t}$ é a variável dependente. Então, em outras palavras, tem-se o preço da ação no período anterior como variável independente e o preço da ação no período atual como variável dependente. O software STATA será utilizado no procedimento de análise de dados em painel. Da mesma forma que nas etapas anteriores calcula-se o erro. E, por fim, será comparado os erros obtidos pelos três métodos de previsão.

\section{DISCUSSÃO DOS RESULTADOS}

A Tabela 1 mostra os resultados parciais obtidos na previsão do preço das ações do ano de 2014, mês a mês para cada empresa integrante da amostra. Vale ressaltar que dois métodos foram utilizados para proceder essa análise, sendo eles: séries temporais - metodologia Box \& Jenkins e análise de dados em painel.

Tabela 1 - Resultados da previsão do preço das ações

\begin{tabular}{|c|c|c|c|c|c|c|}
\hline & BRADESCO & $\begin{array}{c}\text { Séries } \\
\text { Temporais }\end{array}$ & $\begin{array}{c}\text { Dados em } \\
\text { Painel }\end{array}$ & $\begin{array}{l}\text { BRADES- } \\
\text { PAR }\end{array}$ & $\begin{array}{c}\text { Séries } \\
\text { Temporais }\end{array}$ & $\begin{array}{c}\text { Dados em } \\
\text { Painel }\end{array}$ \\
\hline $\begin{array}{l}\text { Mês/ } \\
2014\end{array}$ & $\begin{array}{c}\text { Preço } \\
\text { Observado }\end{array}$ & $\begin{array}{c}\text { Preço } \\
\text { Previsto }\end{array}$ & $\begin{array}{c}\text { Preço } \\
\text { Previsto }\end{array}$ & $\begin{array}{c}\text { Preço } \\
\text { Observado }\end{array}$ & $\begin{array}{c}\text { Preço } \\
\text { Previsto }\end{array}$ & $\begin{array}{c}\text { Preço } \\
\text { Previsto }\end{array}$ \\
\hline Jane & 0,1286 & 0,14331 & 0,12865 & 0,10927 & 0,12280 & 0,10959 \\
\hline Fev & 0,1344 & 0,12862 & 0,13433 & 0,10431 & 0,10583 & 0,10470 \\
\hline Mar & 0,1538 & 0,13439 & 0,15349 & 0,09614 & 0,10308 & 0,09665 \\
\hline Abr & 0,1639 & 0,15384 & 0,16337 & 0,09424 & 0,09412 & 0,09477 \\
\hline Maio & 0,1539 & 0,16386 & 0,15359 & 0,09073 & 0,09376 & 0,09131 \\
\hline Jun & 0,1581 & 0,15394 & 0,15774 & 0,09900 & 0,08986 & 0,09946 \\
\hline Jul & 0,1710 & 0,15815 & 0,17038 & 0,11278 & 0,10104 & 0,11304 \\
\hline Ago & 0,2022 & 0,17098 & 0,20115 & 0,10000 & 0,11619 & 0,10045 \\
\hline Set & 0,1721 & 0,20221 & 0,17152 & 0,08732 & 0,09684 & 0,08796 \\
\hline Out & 0,1846 & 0,17213 & 0,18376 & 0,08140 & 0,08418 & 0,08213 \\
\hline Nov & 0,1958 & 0,18456 & 0,19483 & 0,07308 & 0,07994 & 0,07393 \\
\hline Dez & 0,1732 & 0,19579 & 0,17260 & 0,06917 & 0,07102 & 0,07008 \\
\hline \multicolumn{2}{|c|}{ Erro quadrático médio } & 0,0003064765 & 0,0000003718 & - & 0,0000729132 & 0,0000003407 \\
\hline & BRASIL & $\begin{array}{c}\text { Séries } \\
\text { Temporais }\end{array}$ & $\begin{array}{c}\text { Dados em } \\
\text { Painel } \\
\end{array}$ & BRASKEM & $\begin{array}{c}\text { Séries } \\
\text { Temporais }\end{array}$ & $\begin{array}{c}\text { Dados em } \\
\text { Painel } \\
\end{array}$ \\
\hline $\begin{array}{l}\text { Mês/ } \\
2014\end{array}$ & $\begin{array}{c}\text { Preço } \\
\text { Observado }\end{array}$ & $\begin{array}{c}\text { Preço } \\
\text { Previsto }\end{array}$ & $\begin{array}{c}\text { Preço } \\
\text { Previsto }\end{array}$ & $\begin{array}{c}\text { Preço } \\
\text { Observado }\end{array}$ & $\begin{array}{c}\text { Preço } \\
\text { Previsto }\end{array}$ & $\begin{array}{c}\text { Preço } \\
\text { Previsto }\end{array}$ \\
\hline Jane & 0,10221 & 0,11980 & 0,10262 & 0,06962 & 0,08020 & 0,07052 \\
\hline Fev & 0,10125 & 0,10221 & 0,10168 & 0,06251 & 0,06962 & 0,06351 \\
\hline Mar & 0,11178 & 0,10125 & 0,11206 & 0,06757 & 0,06251 & 0,06850 \\
\hline Abr & 0,11484 & 0,11178 & 0,11507 & 0,05764 & 0,06757 & 0,05872 \\
\hline Maio & 0,11163 & 0,11484 & 0,11191 & 0,05694 & 0,05764 & 0,05803 \\
\hline
\end{tabular}




\begin{tabular}{|c|c|c|c|c|c|c|}
\hline Jun & 0,12206 & 0,11163 & 0,12218 & 0,05388 & 0,05694 & 0,05501 \\
\hline Jul & 0,13644 & 0,12206 & 0,13635 & 0,05153 & 0,05388 & 0,05269 \\
\hline Ago & 0,17288 & 0,13644 & 0,17226 & 0,05464 & 0,05153 & 0,05576 \\
\hline Set & 0,12431 & 0,17288 & 0,12440 & 0,05614 & 0,05464 & 0,05724 \\
\hline Out & 0,13649 & 0,12431 & 0,13640 & 0,06401 & 0,05614 & 0,06499 \\
\hline Nov & 0,14612 & 0,13649 & 0,14588 & 0,06486 & 0,06401 & 0,06583 \\
\hline Dez & 0,11664 & 0,14612 & 0,11685 & 0,05163 & 0,06486 & 0,05279 \\
\hline \multicolumn{2}{|c|}{ Erro quadrático médio } & 0,0004627817 & 0,0000000910 & - & 0,0000459783 & 0,0000011125 \\
\hline & BRF S/A & $\begin{array}{c}\text { Séries } \\
\text { Temporais }\end{array}$ & $\begin{array}{c}\text { Dados em } \\
\text { Painel }\end{array}$ & CCR S/A & $\begin{array}{c}\text { Séries } \\
\text { Temporais }\end{array}$ & $\begin{array}{c}\text { Dados em } \\
\text { Painel }\end{array}$ \\
\hline $\begin{array}{l}\text { Mês/ } \\
2014 \\
\end{array}$ & $\begin{array}{c}\text { Preço } \\
\text { Observado }\end{array}$ & $\begin{array}{c}\text { Preço } \\
\text { Previsto }\end{array}$ & $\begin{array}{c}\text { Preço } \\
\text { Previsto }\end{array}$ & $\begin{array}{c}\text { Preço } \\
\text { Observado }\end{array}$ & $\begin{array}{c}\text { Preço } \\
\text { Previsto }\end{array}$ & $\begin{array}{c}\text { Preço } \\
\text { Previsto }\end{array}$ \\
\hline Jane & 0,21424 & 0,24436 & 0,21300 & 0,07539 & 0,08657 & 0,07620 \\
\hline Fev & 0,21263 & 0,21424 & 0,21142 & 0,07810 & 0,07539 & 0,07887 \\
\hline Mar & 0,22456 & 0,21263 & 0,22317 & 0,08481 & 0,07810 & 0,08549 \\
\hline Abr & 0,25063 & 0,22456 & 0,24886 & 0,08496 & 0,08481 & 0,08563 \\
\hline Maio & 0,23915 & 0,25063 & 0,23755 & 0,08556 & 0,08496 & 0,08623 \\
\hline Jun & 0,26516 & 0,23915 & 0,26318 & 0,08772 & 0,08556 & 0,08835 \\
\hline Jul & 0,27569 & 0,26516 & 0,27355 & 0,08692 & 0,08772 & 0,08756 \\
\hline Ago & 0,29774 & 0,27569 & 0,29528 & 0,09905 & 0,08692 & 0,09951 \\
\hline Set & 0,28942 & 0,29774 & 0,28708 & 0,08160 & 0,09905 & 0,08233 \\
\hline Out & 0,32075 & 0,28942 & 0,31795 & 0,08997 & 0,08160 & 0,09057 \\
\hline Nov & 0,33033 & 0,32075 & 0,32738 & 0,08596 & 0,08997 & 0,08662 \\
\hline Dez & 0,31549 & 0,33033 & 0,31276 & 0,07474 & 0,08596 & 0,07556 \\
\hline \multicolumn{2}{|c|}{ Erro quadrático médio } & 0,0003750075 & 0,0000045616 & - & 0,0000705589 & 0,0000004681 \\
\hline & CEMIG & $\begin{array}{c}\text { Séries } \\
\text { Temporais }\end{array}$ & $\begin{array}{c}\text { Dados em } \\
\text { Painel } \\
\end{array}$ & $\begin{array}{c}\text { CIA } \\
\text { HERING }\end{array}$ & $\begin{array}{c}\text { Séries } \\
\text { Temporais }\end{array}$ & $\begin{array}{c}\text { Dados em } \\
\text { Painel } \\
\end{array}$ \\
\hline $\begin{array}{l}\text { Mês/ } \\
2014\end{array}$ & $\begin{array}{c}\text { Preço } \\
\text { Observado }\end{array}$ & $\begin{array}{c}\text { Preço } \\
\text { Previsto }\end{array}$ & $\begin{array}{c}\text { Preço } \\
\text { Previsto }\end{array}$ & $\begin{array}{c}\text { Preço } \\
\text { Observado }\end{array}$ & $\begin{array}{c}\text { Preço } \\
\text { Previsto }\end{array}$ & $\begin{array}{c}\text { Preço } \\
\text { Previsto }\end{array}$ \\
\hline Jane & 0,06732 & 0,06772 & 0,06825 & 0,13183 & 0,15074 & 0,13181 \\
\hline Fev & 0,06481 & 0,06732 & 0,06578 & 0,12231 & 0,12756 & 0,12243 \\
\hline Mar & 0,07393 & 0,06481 & 0,07477 & 0,13474 & 0,12853 & 0,13467 \\
\hline Abr & 0,08266 & 0,07393 & 0,08336 & 0,11559 & 0,13375 & 0,11581 \\
\hline Maio & 0,07559 & 0,08266 & 0,07640 & 0,10652 & 0,11148 & 0,10687 \\
\hline Jun & 0,07840 & 0,07559 & 0,07916 & 0,10897 & 0,11252 & 0,10929 \\
\hline Jul & 0,09008 & 0,07840 & 0,09067 & 0,10336 & 0,10639 & 0,10376 \\
\hline Ago & 0,09353 & 0,09008 & 0,09408 & 0,13779 & 0,10515 & 0,13769 \\
\hline Set & 0,07263 & 0,09353 & 0,07349 & 0,12181 & 0,14311 & 0,12193 \\
\hline Out & 0,06882 & 0,07263 & 0,06973 & 0,12281 & 0,10703 & 0,12292 \\
\hline Nov & 0,06807 & 0,06882 & 0,06899 & 0,12005 & 0,13888 & 0,12020 \\
\hline Dez & 0,06336 & 0,06807 & 0,06435 & 0,09900 & 0,10416 & 0,09946 \\
\hline \multicolumn{2}{|c|}{ Erro quadrático médio } & 0,0000705100 & 0,0000006907 & - & 0,0002458164 & 0,0000000612 \\
\hline & GERDAU & $\begin{array}{c}\text { Séries } \\
\text { Temporais }\end{array}$ & $\begin{array}{c}\text { Dados em } \\
\text { Painel } \\
\end{array}$ & ITAUSA & $\begin{array}{c}\text { Séries } \\
\text { Temporais }\end{array}$ & $\begin{array}{c}\text { Dados em } \\
\text { Painel } \\
\end{array}$ \\
\hline $\begin{array}{l}\text { Mês/ } \\
2014\end{array}$ & $\begin{array}{c}\text { Preço } \\
\text { Observado }\end{array}$ & $\begin{array}{c}\text { Preço } \\
\text { Previsto }\end{array}$ & $\begin{array}{c}\text { Preço } \\
\text { Previsto }\end{array}$ & $\begin{array}{c}\text { Preço } \\
\text { Observado }\end{array}$ & $\begin{array}{c}\text { Preço } \\
\text { Previsto }\end{array}$ & $\begin{array}{c}\text { Preço } \\
\text { Previsto }\end{array}$ \\
\hline Jane & 0,08346 & 0,08971 & 0,08415 & 0,13549 & 0,14035 & 0,13542 \\
\hline Fev & 0,06987 & 0,08244 & 0,07077 & 0,13980 & 0,13549 & 0,13966 \\
\hline Mar & 0,07028 & 0,06755 & 0,07116 & 0,15198 & 0,13980 & 0,15166 \\
\hline Abr & 0,06451 & 0,07034 & 0,06548 & 0,16516 & 0,15198 & 0,16465 \\
\hline Maio & 0,06431 & 0,06353 & 0,06529 & 0,15654 & 0,16516 & 0,15616 \\
\hline Jun & 0,06236 & 0,06428 & 0,06336 & 0,15774 & 0,15654 & 0,15734 \\
\hline Jul & 0,06441 & 0,06202 & 0,06539 & 0,17343 & 0,15774 & 0,17280 \\
\hline Ago & 0,06246 & 0,06476 & 0,06346 & 0,19980 & 0,17343 & 0,19878 \\
\hline Set & 0,05654 & 0,06212 & 0,05763 & 0,16727 & 0,19980 & 0,16673 \\
\hline Out & 0,05308 & 0,05553 & 0,05422 & 0,18170 & 0,16727 & 0,18095 \\
\hline Nov & 0,05213 & 0,05249 & 0,05329 & 0,19098 & 0,18170 & 0,19009 \\
\hline
\end{tabular}




\begin{tabular}{|c|c|c|c|c|c|c|}
\hline Dez & 0,04551 & 0,05197 & 0,04677 & 0,17093 & 0,19098 & 0,17033 \\
\hline \multicolumn{2}{|c|}{ Erro quadrático médio } & 0,0000277242 & 0,0000010286 & - & 0,0002613462 & 0,0000003463 \\
\hline & $\begin{array}{l}\text { ITAUUNI- } \\
\text { BANCO }\end{array}$ & $\begin{array}{c}\text { Séries } \\
\text { Temporais }\end{array}$ & $\begin{array}{c}\text { Dados em } \\
\text { Painel }\end{array}$ & $\begin{array}{l}\text { KLABIN } \\
\text { S/A }\end{array}$ & $\begin{array}{c}\text { Séries } \\
\text { Temporais }\end{array}$ & $\begin{array}{c}\text { Dados em } \\
\text { Painel }\end{array}$ \\
\hline $\begin{array}{l}\text { Mês/ } \\
2014\end{array}$ & $\begin{array}{c}\text { Preço } \\
\text { Observado }\end{array}$ & $\begin{array}{c}\text { Preço } \\
\text { Previsto }\end{array}$ & $\begin{array}{c}\text { Preço } \\
\text { Previsto }\end{array}$ & $\begin{array}{c}\text { Preço } \\
\text { Observado }\end{array}$ & $\begin{array}{c}\text { Preço } \\
\text { Previsto }\end{array}$ & $\begin{array}{c}\text { Preço } \\
\text { Previsto }\end{array}$ \\
\hline Jane & 0,03584 & 0,03805 & 0,03724 & 0,00987 & 0,00980 & 0,01165 \\
\hline Fev & 0,03619 & 0,03584 & 0,03758 & 0,00912 & 0,00989 & 0,01091 \\
\hline Mar & 0,03965 & 0,03619 & 0,04099 & 0,00917 & 0,00898 & 0,01096 \\
\hline Abr & 0,04216 & 0,03965 & 0,04346 & 0,00902 & 0,00918 & 0,01081 \\
\hline Maio & 0,04030 & 0,04216 & 0,04163 & 0,00847 & 0,00899 & 0,01027 \\
\hline Jun & 0,04105 & 0,04030 & 0,04237 & 0,00847 & 0,00837 & 0,01027 \\
\hline Jul & 0,04506 & 0,04105 & 0,04632 & 0,00877 & 0,00847 & 0,01057 \\
\hline Ago & 0,05213 & 0,04506 & 0,05329 & 0,00882 & 0,00883 & 0,01062 \\
\hline Set & 0,04396 & 0,05213 & 0,04524 & 0,00937 & 0,00883 & 0,01116 \\
\hline Out & 0,04707 & 0,04396 & 0,04830 & 0,00962 & 0,00948 & 0,01141 \\
\hline Nov & 0,05003 & 0,04707 & 0,05121 & 0,01098 & 0,00967 & 0,01274 \\
\hline Dez & 0,04456 & 0,05003 & 0,04583 & 0,01193 & 0,01123 & 0,01368 \\
\hline \multicolumn{2}{|c|}{ Erro quadrático médio } & 0,0000173567 & 0,0000016647 & - & 0,0000002950 & 0,0000031876 \\
\hline & $\begin{array}{c}\text { LOJAS } \\
\text { AMERIC } \\
\end{array}$ & $\begin{array}{c}\text { Séries } \\
\text { Temporais }\end{array}$ & $\begin{array}{c}\text { Dados em } \\
\text { Painel }\end{array}$ & OI & $\begin{array}{c}\text { Séries } \\
\text { Temporais }\end{array}$ & $\begin{array}{c}\text { Dados em } \\
\text { Painel } \\
\end{array}$ \\
\hline $\begin{array}{l}\text { Mês/ } \\
2014 \\
\end{array}$ & $\begin{array}{c}\text { Preço } \\
\text { Observado }\end{array}$ & $\begin{array}{c}\text { Preço } \\
\text { Previsto } \\
\end{array}$ & $\begin{array}{c}\text { Preço } \\
\text { Previsto } \\
\end{array}$ & $\begin{array}{c}\text { Preço } \\
\text { Observado }\end{array}$ & $\begin{array}{c}\text { Preço } \\
\text { Previsto }\end{array}$ & $\begin{array}{c}\text { Preço } \\
\text { Previsto } \\
\end{array}$ \\
\hline Jane & 0,05644 & 0,06055 & 0,05753 & 0,20652 & 0,17744 & 0,20539 \\
\hline Fev & 0,05554 & 0,05644 & 0,05664 & 0,17744 & 0,20652 & 0,17675 \\
\hline Mar & 0,06456 & 0,05554 & 0,06553 & 0,15389 & 0,17744 & 0,15354 \\
\hline Abr & 0,06521 & 0,06456 & 0,06618 & 0,10476 & 0,15389 & 0,10514 \\
\hline Maio & 0,06411 & 0,06521 & 0,06509 & 0,09474 & 0,10476 & 0,09526 \\
\hline Jun & 0,06817 & 0,06411 & 0,06909 & 0,09524 & 0,09474 & 0,09576 \\
\hline Jul & 0,06992 & 0,06817 & 0,07082 & 0,07118 & 0,09524 & 0,07205 \\
\hline Ago & 0,07689 & 0,06992 & 0,07768 & 0,07018 & 0,07118 & 0,07107 \\
\hline Set & 0,06712 & 0,07689 & 0,06805 & 0,08471 & 0,07018 & 0,08539 \\
\hline Out & 0,07078 & 0,06712 & 0,07166 & 0,06266 & 0,08471 & 0,06366 \\
\hline Nov & 0,08231 & 0,07078 & 0,08302 & 0,06667 & 0,06266 & 0,06761 \\
\hline Dez & 0,08391 & 0,08231 & 0,08460 & 0,04065 & 0,06667 & 0,04198 \\
\hline \multicolumn{2}{|c|}{ Erro quadrático médio } & 0,0005608129 & 0,0000008456 & - & 0,0000344399 & 0,0000006840 \\
\hline & $\begin{array}{l}\text { PETRO- } \\
\text { BRAS }\end{array}$ & $\begin{array}{c}\text { Séries } \\
\text { Temporais }\end{array}$ & $\begin{array}{c}\text { Dados em } \\
\text { Painel }\end{array}$ & SABESP & $\begin{array}{c}\text { Séries } \\
\text { Temporais }\end{array}$ & $\begin{array}{c}\text { Dados em } \\
\text { Painel }\end{array}$ \\
\hline $\begin{array}{l}\text { Mês/ } \\
2014\end{array}$ & $\begin{array}{c}\text { Preço } \\
\text { Observado }\end{array}$ & $\begin{array}{c}\text { Preço } \\
\text { Previsto }\end{array}$ & $\begin{array}{c}\text { Preço } \\
\text { Previsto }\end{array}$ & $\begin{array}{c}\text { Preço } \\
\text { Observado }\end{array}$ & $\begin{array}{c}\text { Preço } \\
\text { Previsto }\end{array}$ & $\begin{array}{c}\text { Preço } \\
\text { Previsto }\end{array}$ \\
\hline Jane & 0,06652 & 0,07764 & 0,06746 & 0,05358 & 0,07338 & 0,05472 \\
\hline Fev & 0,06261 & 0,06652 & 0,06361 & 0,04877 & 0,04867 & 0,04998 \\
\hline Mar & 0,07248 & 0,06261 & 0,07334 & 0,04682 & 0,04729 & 0,04805 \\
\hline Abr & 0,07584 & 0,07248 & 0,07665 & 0,04040 & 0,04622 & 0,04173 \\
\hline Maio & 0,07639 & 0,07584 & 0,07719 & 0,04055 & 0,03843 & 0,04188 \\
\hline Jun & 0,07890 & 0,07639 & 0,07966 & 0,04461 & 0,04060 & 0,04588 \\
\hline Jul & 0,08767 & 0,07890 & 0,08830 & 0,05489 & 0,04586 & 0,05600 \\
\hline Ago & 0,10847 & 0,08767 & 0,10880 & 0,04672 & 0,05804 & 0,04795 \\
\hline Set & 0,08396 & 0,10847 & 0,08465 & 0,04110 & 0,04421 & 0,04242 \\
\hline Out & 0,07093 & 0,08396 & 0,07181 & 0,03885 & 0,03938 & 0,04020 \\
\hline Nov & 0,05840 & 0,07093 & 0,05946 & 0,02787 & 0,03815 & 0,02938 \\
\hline Dez & 0,04556 & 0,05840 & 0,04682 & 0,02546 & 0,02450 & 0,02701 \\
\hline \multicolumn{2}{|c|}{ Erro quadrático médio } & $\mathbf{0 , 0 0 0 1 5 4 7 0 8 9}$ & $\mathbf{0 , 0 0 0 0 0 0 7 4 5 3}$ & - & 0,0000644103 & 0,0000017024 \\
\hline & $\begin{array}{c}\text { SID } \\
\text { NACIONAL }\end{array}$ & $\begin{array}{c}\text { Séries } \\
\text { Temporais }\end{array}$ & $\begin{array}{c}\text { Dados em } \\
\text { Painel } \\
\end{array}$ & $\begin{array}{c}\text { SOUZA } \\
\text { CRUZ }\end{array}$ & $\begin{array}{c}\text { Séries } \\
\text { Temporais }\end{array}$ & $\begin{array}{c}\text { Dados em } \\
\text { Painel } \\
\end{array}$ \\
\hline $\begin{array}{l}\text { Mês/ } \\
2014 \\
\end{array}$ & $\begin{array}{c}\text { Preço } \\
\text { Observado }\end{array}$ & $\begin{array}{c}\text { Preço } \\
\text { Previsto } \\
\end{array}$ & $\begin{array}{c}\text { Preço } \\
\text { Previsto } \\
\end{array}$ & $\begin{array}{c}\text { Preço } \\
\text { Observado } \\
\end{array}$ & $\begin{array}{c}\text { Preço } \\
\text { Previsto } \\
\end{array}$ & $\begin{array}{c}\text { Preço } \\
\text { Previsto } \\
\end{array}$ \\
\hline
\end{tabular}




\begin{tabular}{|c|c|c|c|c|c|c|}
\hline Jane & 0,10907 & 0,13013 & 0,10939 & 0,10381 & $-0,00040$ & 0,10381 \\
\hline Fev & 0,10526 & 0,10907 & 0,10564 & 0,09991 & $-0,00046$ & 0,09991 \\
\hline Mar & 0,10326 & 0,10526 & 0,10366 & 0,10119 & $-0,00044$ & 0,10119 \\
\hline Abr & 0,10336 & 0,10326 & 0,10376 & 0,09996 & $-0,00046$ & 0,09996 \\
\hline Maio & 0,10667 & 0,10336 & 0,10702 & 0,11280 & $-0,00027$ & 0,11280 \\
\hline Jun & 0,11554 & 0,10667 & 0,11576 & 0,11191 & $-0,00028$ & 0,11191 \\
\hline Jul & 0,09820 & 0,11554 & 0,09867 & 0,10346 & $-0,00040$ & 0,10346 \\
\hline Ago & 0,10471 & 0,09820 & 0,10509 & 0,10297 & $-0,00041$ & 0,10297 \\
\hline Set & 0,09664 & 0,10471 & 0,09714 & 0,09675 & $-0,00051$ & 0,09675 \\
\hline Out & 0,09469 & 0,09664 & 0,09522 & 0,09833 & $-0,00048$ & 0,09833 \\
\hline Nov & 0,09378 & 0,09469 & 0,09433 & 0,10070 & $-0,00045$ & 0,10070 \\
\hline Dez & 0,08276 & 0,09378 & 0,08346 & 0,09487 & $-0,00053$ & 0,09487 \\
\hline \multicolumn{2}{|c|}{ Erro quadrático médio } & $\mathbf{0 , 0 0 0 0 9 0 5 0 3 5}$ & 0,0000002019 & - & 0,0000001854 & 0,0000001854 \\
\hline & $\begin{array}{l}\text { SUZANO } \\
\text { PAPEL }\end{array}$ & $\begin{array}{c}\text { Séries } \\
\text { Temporais }\end{array}$ & $\begin{array}{c}\text { Dados em } \\
\text { Painel } \\
\end{array}$ & $\begin{array}{r}\text { TELEF } \\
\text { BRASIL } \\
\end{array}$ & $\begin{array}{c}\text { Séries } \\
\text { Temporais }\end{array}$ & $\begin{array}{c}\text { Dados em } \\
\text { Painel } \\
\end{array}$ \\
\hline $\begin{array}{l}\text { Mês/ } \\
2014 \\
\end{array}$ & $\begin{array}{c}\text { Preço } \\
\text { Observado }\end{array}$ & $\begin{array}{c}\text { Preço } \\
\text { Previsto }\end{array}$ & $\begin{array}{c}\text { Preço } \\
\text { Previsto } \\
\end{array}$ & $\begin{array}{c}\text { Preço } \\
\text { Observado }\end{array}$ & $\begin{array}{c}\text { Preço } \\
\text { Previsto } \\
\end{array}$ & $\begin{array}{c}\text { Preço } \\
\text { Previsto } \\
\end{array}$ \\
\hline Jane & 0,04521 & 0,04458 & 0,04647 & 0,22742 & 0,22221 & 0,22599 \\
\hline Fev & 0,04201 & 0,04551 & 0,04331 & 0,21293 & 0,22742 & 0,21172 \\
\hline Mar & 0,03950 & 0,04134 & 0,04084 & 0,23825 & 0,21293 & 0,23666 \\
\hline Abr & 0,03393 & 0,03898 & 0,03536 & 0,23228 & 0,23825 & 0,23078 \\
\hline Maio & 0,03794 & 0,03278 & 0,03931 & 0,22181 & 0,23228 & 0,22046 \\
\hline Jun & 0,03955 & 0,03878 & 0,04089 & 0,22306 & 0,22181 & 0,22169 \\
\hline Jul & 0,04160 & 0,03988 & 0,04292 & 0,22607 & 0,22306 & 0,22466 \\
\hline Ago & 0,04216 & 0,04203 & 0,04346 & 0,23734 & 0,22607 & 0,23577 \\
\hline Set & 0,04682 & 0,04227 & 0,04805 & 0,24010 & 0,23734 & 0,23848 \\
\hline Out & 0,04992 & 0,04779 & 0,05111 & 0,25118 & 0,24010 & 0,24940 \\
\hline Nov & 0,05203 & 0,05057 & 0,05319 & 0,26070 & 0,25118 & 0,25878 \\
\hline Dez & 0,05388 & 0,05247 & 0,05501 & 0,23233 & 0,26070 & 0,23083 \\
\hline \multicolumn{2}{|c|}{ Erro quadrático médio } & 0,0000084258 & 0,0000016462 & - & $\mathbf{0 , 0 0 0 1 8 2 2 3 8 3}$ & 0,0000023476 \\
\hline & $\begin{array}{c}\text { TIM PART } \\
\text { S/A } \\
\end{array}$ & $\begin{array}{c}\text { Séries } \\
\text { Temporais }\end{array}$ & $\begin{array}{c}\text { Dados em } \\
\text { Painel } \\
\end{array}$ & USIMINAS & $\begin{array}{c}\text { Séries } \\
\text { Temporais }\end{array}$ & $\begin{array}{c}\text { Dados em } \\
\text { Painel } \\
\end{array}$ \\
\hline $\begin{array}{l}\text { Mês/ } \\
2014 \\
\end{array}$ & $\begin{array}{c}\text { Preço } \\
\text { Observado }\end{array}$ & $\begin{array}{c}\text { Preço } \\
\text { Previsto } \\
\end{array}$ & $\begin{array}{c}\text { Preço } \\
\text { Previsto }\end{array}$ & $\begin{array}{c}\text { Preço } \\
\text { Observado }\end{array}$ & $\begin{array}{c}\text { Preço } \\
\text { Previsto } \\
\end{array}$ & $\begin{array}{c}\text { Preço } \\
\text { Previsto } \\
\end{array}$ \\
\hline Jane & 0,06085 & 0,05930 & 0,06188 & 0,05714 & 0,07166 & 0,05823 \\
\hline Fev & 0,05494 & 0,06085 & 0,05605 & 0,04647 & 0,05328 & 0,04771 \\
\hline Mar & 0,05689 & 0,05494 & 0,05798 & 0,04877 & 0,04291 & 0,04998 \\
\hline Abr & 0,05815 & 0,05689 & 0,05921 & 0,04120 & 0,04954 & 0,04252 \\
\hline Maio & 0,05845 & 0,05815 & 0,05951 & 0,03704 & 0,03868 & 0,03842 \\
\hline Jun & 0,06226 & 0,05845 & 0,06326 & 0,03549 & 0,03566 & 0,03689 \\
\hline Jul & 0,05774 & 0,06226 & 0,05882 & 0,03784 & 0,03497 & 0,03921 \\
\hline Ago & 0,06040 & 0,05774 & 0,06144 & 0,03800 & 0,03863 & 0,03936 \\
\hline Set & 0,06211 & 0,06040 & 0,06311 & 0,02942 & 0,03805 & 0,03091 \\
\hline Out & 0,06501 & 0,06211 & 0,06598 & 0,02607 & 0,02657 & 0,02761 \\
\hline Nov & 0,05995 & 0,06501 & 0,06099 & 0,02356 & 0,02495 & 0,02514 \\
\hline Dez & 0,05654 & 0,05995 & 0,05763 & 0,02281 & 0,02272 & 0,02440 \\
\hline \multicolumn{2}{|c|}{ Erro quadrático médio } & 0,0000111174 & 0,0000011005 & - & 0,0000000000 & 0,0000000000 \\
\hline & VALE & $\begin{array}{c}\text { Séries } \\
\text { Temporais }\end{array}$ & $\begin{array}{c}\begin{array}{c}\text { Dados em } \\
\text { Painel }\end{array} \\
\end{array}$ & & & \\
\hline $\begin{array}{l}\text { Mês/ } \\
2014 \\
\end{array}$ & $\begin{array}{c}\text { Preço } \\
\text { Observado }\end{array}$ & $\begin{array}{c}\text { Preço } \\
\text { Previsto } \\
\end{array}$ & $\begin{array}{c}\text { Preço } \\
\text { Previsto } \\
\end{array}$ & & & \\
\hline Jane & 0,14787 & 0,16150 & 0,14761 & & & \\
\hline Fev & 0,14331 & 0,14518 & 0,14312 & & & \\
\hline Mar & 0,13960 & 0,14241 & 0,13946 & & & \\
\hline Abr & 0,12993 & 0,13887 & 0,12993 & & & \\
\hline Maio & 0,12602 & 0,12802 & 0,12608 & & & \\
\hline Jun & 0,12957 & 0,12525 & 0,12959 & & & \\
\hline
\end{tabular}




\begin{tabular}{c|c|c|c} 
Jul & 0,14351 & 0,13027 & 0,14332 \\
\hline Ago & 0,12767 & 0,14625 & 0,12771 \\
\hline Set & 0,11669 & 0,12456 & 0,11690 \\
\hline Out & 0,10551 & 0,11454 & 0,10588 \\
\hline Nov & 0,09774 & 0,10332 & 0,09823 \\
\hline Dez & 0,09388 & 0,09622 & 0,09442 \\
\hline Erro quadrático médio & $\mathbf{0 , 0 0 0 0 8 3 3 2 4 0}$ & $\mathbf{0 , 0 0 0 0 0 0 0 7 2 1}$ \\
\hline
\end{tabular}

Destaca-se que para a análise de séries temporais da empresa BRADESCO, o modelo mais adequado foi o ARIMA $(0,1,0)$ e o erro quadrático médio foi de 0,0003064765 . Por sua vez, a análise de dados em painel evidenciou um erro quadrático médio de 0,0000003718. Logo, para esta empresa, conclui-se que a previsão multivariada apresentou um erro inferior à previsão feita por séries temporais.

No caso da empresa BRADESPAR o modelo de séries temporais que se mostrou mais adequado é o ARIMA $(1,1,0)$ e o erro quadrático médio é de 0,0000729132 . Nesse mesmo sentido, a análise de dados em painel forneceu um erro quadrático de 0,0000003407. Portanto, verifica-se que a modelagem multivariada apresentou melhores previsões do que o modelo univariado.

Para a empresa BRASIL, o melhor modelo de séries temporais é o ARIMA $(0,1,0)$ e o erro quadrático médio é 0,0004627817 . No que tange os resultados encontrados pela análise de dados em painel, o erro quadrático médio é de 0,0000000910. Dessa forma, o erro quadrático médio obtido pela análise de dados em painel é menor do que o obtido por séries temporais.

Em relação a empresa BRASKEM, verifica-se que o modelo mais adequado para a análise de séries temporais é o ARIMA $(0,1,0)$ e o erro quadrático médio é 0,0000459783 . Por sua vez, o erro quadrático médio obtido com a análise de dados em painel foi melhor, 0,0000011125 , e dessa forma contata-se que para esta empresa, o modelo multivariado foi mais adequado para a previsão.

De acordo com a análise feita utilizando o método de séries temporais o melhor modelo para a empresa BRF S/A é o ARIMA $(0,1,0)$ e o erro quadrático médio que diz respeito a esse modelo é 0,0003750075 . Por outro lado, no que tange os resultados obtidos pela análise de dados em painel obteve-se um erro quadrático médio de 0,0000045616. Dessa forma, percebese que uma vez mais o modelo de dados em painel foi mais assertivo nas previsões em relação ao de séries temporais.

Conforme resultados obtidos com a análise de séries temporais para a empresa CCR S/A, o modelo que melhor se adequa a esta série de dados é o $\operatorname{ARIMA~}(0,1,0)$ e o erro quadrático médio encontrado é de 0,0000705589 . Na sequência procedeu-se a análise por dados em painel e obteve-se um erro quadrático médio de 0,0000004681. Ou seja, é fácil verificar que o modelo de análise por dados em painel evidencia melhores previsões.

Conforme exposto na Tabela 1, no que tange a empresa CEMIG, o erro quadrático médio obtido pela análise univariada de séries temporais é de 0,0000705100 e o modelo mais adequado é o ARIMA $(0,1,0)$. Em contrapartida, o erro quadrático médio obtido com a análise de dados em painel é de 0,0000006907. Logo, a segunda análise apresentou resultados mais precisos em relação as previsões.

De acordo com a análise de séries temporais realizada, o melhor modelo para a empresa CIA HERING é o ARIMA $(1,1,2)$ com erro quadrático médio respectivo de 0,0002458164 . Nesse mesmo sentido, a análise realizada pelo método de dados em painel mostrou um erro quadrático médio inferior, sendo de 0,0000000612, que caracteriza que estes resultados são mais precisos do que os obtidos com o modelo univariado.

Destaca-se que o modelo que mais se adequa a série de tempo da empresa GERDAU é o ARIMA $(1,1,0)$ e seu erro quadrático médio encontrado em relação a precisão das previsões 
é de 0,0000277242 . De forma análoga procedeu-se a previsão utilizando o modelo de dados em painel e o erro quadrático médio encontrado foi de 0,0000010286 . Ou seja, o segundo modelo se mostrou mais preciso em relação ao primeiro.

No caso da empresa ITAUSA o melhor modelo para as previsões obtidas a partir da análise da série temporal é o ARIMA $(0,1,0)$ e seu erro quadrático médio é de 0,0002613462 . Já em relação a análise por meio de dados em painel obteve-se um erro quadrático médio de 0,0000003463, mais uma vez inferior a análise anterior se mostrando mais preciso.

De acordo com a análise univariada de séries temporais o melhor modelo para os dados referentes a empresa ITAUUNIBANCO é o ARIMA $(0,1,0)$ e o erro quadrático médio encontrado é de 0,0000173567 . Por outro lado, a análise de dados em painel evidenciou um erro quadrático médio de 0,0000016647 . Dessa forma, verifica-se que neste caso o modelo multivariado também se mostrou mais preciso.

Para esta série de dados de tempo da empresa KLABIN S/A, o modelo mais adequado é o ARIMA $(1,1,0)$ e seu respectivo erro quadrático médio é de 0,0000002950 . Por sua vez, a análise de dados em painel evidenciou um erro quadrático médio de 0,0000031876. Neste caso, a análise dos dados por meio de séries temporais apresentou melhor resultado em relação a análise multivariada.

A análise realizada por meio de séries temporais evidenciou que o melhor modelo para a empresa LOJAS AMERIC é o ARIMA $(0,1,0)$ e seu respectivo erro quadrático médio é de 0,0005608129. Na sequência procedeu-se a análise por meio de dados em painel e o erro quadrático médio encontrado foi de 0,0000008456 . Logo, o modelo mais preciso para os dados referentes a esta empresa é de dados em painel.

Conforme os resultados obtidos com a análise dos dados por meio de séries temporais o melhor modelo para os dados da empresa OI é o ARIMA $(0,1,0)$ e o erro quadrático médio é de 0,0000344399 . Já para a análise que utilizou o modelo de dados em painel o erro quadrático médio é de 0,0000006840, e nesse caso este segundo modelo se mostrou mais preciso em detrimento ao primeiro.

Neste caso, a análise dos dados realizada por meio de análise de séries temporais para a empresa PETROBRAS mostra que o modelo mais adequado é o ARIMA $(0,1,0)$ e seu respectivo erro quadrático médio é de 0,0001547089 . No que tange a análise realizada com o modelo de dados em painel, encontrou-se um erro quadrático médio de 0,0000007453 . Como o erro obtido com o segundo modelo é inferior ao obtido com o primeiro, diz-se que o segundo foi mais preciso.

O modelo ARIMA $(1,1,0)$ se mostrou mais adequado ao conjunto de dados referente a empresa SABESP, em relação a análise de séries temporais, e o erro quadrático médio encontrado é de 0,0000644103. Nesse mesmo sentido, a análise que utiliza dados em painel evidenciou um erro quadrático médio de 0,0000017024 , e portanto apresenta-se mais preciso.

A análise que utilizou a técnica de séries temporais resultou no modelo ARIMA $(0,1,0)$ como o mais adequado para a empresa SID NACIONAL, e respectivo erro quadrático médio de 0,0000905035. De forma similar a análise de dados em painel resultou num erro quadrático médio de 0,0000002019. Uma vez mais, o modelo multivariado se mostra mais preciso em relação ao modelo univariado.

No caso da empresa SOUZA CRUZ, o erro quadrático médio obtido com a análise dos dados por meio de séries temporais foi de 0,0000001854 e o modelo que forneceu esse erro foi o ARIMA $(0,1,0)$. Na sequência, procedeu-se a análise por meio do modelo de dados em painel e o erro quadrático médio encontrado foi de 0,0000001854 . Neste caso, não tem modelo mais ou menos adequado, visto que os erros médios quadráticos são iguais para ambos os modelos.

Para a empresa SUZANO PAPEL, a análise de séries temporais forneceu como modelo mais adequado o ARIMA $(1,1,0)$ e o erro quadrático médio de 0,0000084258 . Enquanto que a 
análise que utilizou dados em painel apresentou um erro quadrático médio de 0,0000016462. Logo, neste caso o modelo de análise de dados em painel se mostrou mais preciso.

O modelo de análise de séries temporais mais adequado para o conjunto de dados da empresa TELEF BRASIL é o ARIMA $(0,1,0)$ com erro quadrático médio de 0,0001822383 . Por outro lado, a análise que utiliza o modelo de dados em painel forneceu um erro quadrático médio de 0,0000023476. Dessa forma, é fácil verificar que novamente o modelo multivariado se mostrou mais adequado em detrimento ao univariado.

O modelo de análise de séries de tempo mais adequado para a empresa TIM PART S/A é o ARIMA $(0,1,0)$ e seu respectivo erro quadrático médio é 0,0000111174 . Na sequência, a análise que utiliza o modelo de dados em painel evidencia um erro quadrático médio de 0,0000011005. Portanto o modelo de dados em painel é mais preciso para estes dados.

De acordo com os resultados expostos para a empresa USIMINAS, ambos os métodos utilizados para realizar a previsão desse conjunto de dados foram bastante eficientes, dado que os dois erros médios quadráticos ficaram zerados. Em outras palavras, tanto a análise de séries temporais, por meio do modelo ARIMA $(1,1,0)$, quanto aquela feita por dados em painel se mostraram precisas iguais.

O modelo de séries temporais mais adequado ao conjunto de dados da empresa VALE é o ARIMA $(0,1,0)$ e seu respectivo erro quadrático médio é 0,0000833240 . Por sua vez, a análise do modelo de dados em painel forneceu um erro quadrático médio de 0,0000000721. Deste modo, o modelo multivariado se apresentou mais preciso em relação ao modelo univariadodos da pesquisa.

A Tabela 2 apresenta os erros médios globais obtidos a partir de cada modelo utilizado para fazer as previsões. Esses erros foram obtidos fazendo a média aritmética entre os erros médios quadráticos obtidos para cada empresa.

Tabela 1 - Erros médios quadráticos globais

\begin{tabular}{c|c}
\hline Métodos & Erro quadrático médio \\
\hline Séries Temporais & 0,0001388791 \\
\hline Dados em Painel & 0,0000010198 \\
\hline
\end{tabular}

Fonte: Dados da pesquisa.

Conforme evidenciado na Tabela 2, o modelo de séries temporais apresenta um erro quadrático médio global de 0,001388791; ao passo que o modelo de dados em painel tem esse erro com valor de 0,0000010198. Sendo assim, é fácil verificar que o modelo mais preciso é, de fato, o multivariado (dados em painel).

Este resultado já era esperado, visto que em apenas 3 das 23 empresas analisadas, o modelo multivariado não teve desempenho superior ao univariado, sendo que em 2 casos houve empate. Ou seja, em $87 \%$ das empresas pesquisadas o modelo de análise de dados em painel previu de forma mais precisa o preço das ações de 2014 (mês a mês).

$\mathrm{O}$ fato de o resultado obtido por meio do modelo multivariado ser melhor do que o modelo univariado corrobora com a ideia de diversificação de Markowitz (1952), pois parece que o modelo multivariado é mais sensível a oscilações. E, com esse resultado a tese deste estudo está comprovada.

\section{CONCLUSÕES}

Este trabalho teve como objetivo geral avaliar qual o melhor método de previsão do preço das ações da carteira teórica composta pelas empresas integrantes do IBrX-50, análise de séries temporais ou análise de dados em painel.

Para tanto utilizou-se 23 empresas que compõem o índice IBRX-50 e que estavam listadas na BM\&FBovespa desde ao menos 2004. A variável analisada é o preço de fechamento 
da ação do último dia útil de cada mês. E a tese do trabalho, está justamente em admitir que uma previsão realizada de forma multivariada seria mais precisa do que as formas univariadas tradicionais de fazer isso. Esta tese tem por base a Teoria de Markowitz (1952) que defende a diversificação das carteiras de ações.

Por fim evidenciou-se que que o método de análise de dados em painel (multivariado) apresentou um desempenho bastante superior ao modelo univariado de séries temporais. Portanto, ao fim, confirma-se parcialmente a tese da pesquisa, de que o modelo multivariado é mais eficiente para prever o preço das ações da carteira teórica composta pelas empresas integrantes do IBRX-50. E, ainda pode-se afirmar que a teoria de Markowitz (1952) que sugere diversificação das carteiras foi comprovada por meio de pesquisa empírica.

\section{REFERÊNCIAS}

ANTUNES, M. A.; PROCIANOY, J. L. Os efeitos das decisões de investimento das empresas sobre os preços de suas ações no mercado de capitais. Revista de Administração, v. 38, n. 1, p. 5-14, 2002.

BOX, G. E. P.; JENKINS, G.; REINSEL, G. C. Time Series Analysis. New Jersey: Prentice Hall, 2008.

BRUNI, A. L. Risco e equilíbrio: uma análise do modelo de precificação de ativos financeiros na avaliação de ações negociadas na Bovespa (1988-1996). 1998. 163 f. Dissertação (mestrado) - Faculdade de Economia, Administração e Contabilidade, Universidade de São Paulo, São Paulo, 1998.

DOWNES, J.; GODMAN, J. E. Dicionário de termos financeiros e de investimento. São Paulo: Nobel, 1993.

DUARTE, P. C.; LAMOUNIER, W. M.; COLAUTO, R. D. Modelos econométricos para dados em painel: aspectos teóricos e exemplos de aplicação à pesquisa em contabilidade e finanças. In: LOPES, J.; RIBEIRO FILHO, J. F.; PEDERNEIRAS, M. (eds.), Educação Contábil: Tópicos de Ensino e Pesquisa. São Paulo, Atlas, p. 256-274, 2008. Disponível em $<$ http://disciplinas.stoa.usp.br/pluginfile.php/176819/mod_resource/content/1/Artigo\%20\%20Modelos\%20e m \%20Painel.pdf>. Acesso em 26/01/15.

EHLERS, R. S. Análise de Séries Temporais. Departamento de Estatística, UFPR,2005. Disponível em < http://www.icmc.usp.br/ ehlers/stemp/stemp.pdf >. Acesso em: 15/01/2015.

FÁVERO, L. P.; BELFIORE, P.; TAKAMATSU, R.T; SUZART, J. Métodos quantitativos com Stata®. Rio de Janeiro: Elsevier, 2014.

GREENE, W. H. Econometric analysis. 6. ed Upper Saddle River: Prentice Hall, 2007.

KRITZMAN, M. What Practitioners Need To Know ... About Duration and Convexity. Financial Analysts Journal, maio/junho, p. 17- 20, 1992.

MAKRIDAKIS, S., WHEELWRIGHT, S. C. \& HYNDMAN, R. J. Forecasting - methods and applications, $3^{\text {a }}$ ed., John Wiley, New York, 1998.

MARKOWITZ, H. Portfolio selection. Journal of Finance, junho, pp. 77 - 91, 1952.

MARQUES, L. D. Modelos dinâmicos com dados em painel: revisão da literatura. Série Working Papers do Centro de Estudos Macroeconômicos e Previsão (CEMPRE) da Faculdade de Economia do Porto, Portugal, $n^{\circ}$ 100, 2000.

MORETTIN, P. A.; TOLOI, C. M. de C. Análise de Séries Temporais. São Paulo: Edgard Blücher, 2006.

SHARPE, W. F.; ALEXANDER, G. J.; BAILEY, J. V. Investments. 5th ed. New Jersey: Prentice-Hall, Englewood, 1995. 